Maarten P. Mulder - Martina Wilke - An Langeveld

Laurens G. Wilming · Anne Hagemeijer

Ellen van Drunen - Ellen C. Zwarthoff

Peter H. J. Riegman - Wout H. Deelen

Ans M. W. van den Ouweland · Dicky J. J. Halley

Carel Meijers

\title{
Positional mapping of loci in the DiGeorge critical region at chromosome 22q11 using a new marker (D22S183)
}

Received: 13 December 1994 / Revised: 4 February 1995

\begin{abstract}
The majority of patients with DiGeorge syndrome (DGS) and velo-cardio-facial syndrome (VCFS) and a minority of patients with non-syndromic conotruncal heart defects are hemizygous for a region of chromosome $22 \mathrm{q} 11$. The chromosomal region that is commonly deleted is larger than $2 \mathrm{Mb}$. It has not been possible to narrow the smallest region of overlap (SRO) of the deletions to less than ca $500 \mathrm{~kb}$, which suggests that DGS/VCFS might be a contiguous gene syndrome. The saturation cloning of the SRO is being carried out, and one gene (TUPLE1) has been identified. By using a cosmid probe (M51) and fluorescence in situ hybridization, we show here that the anonymous DNA marker locus D22S183 is within the SRO, between TUPLE1 and D22S75 (probe N25). A second locus with weak homology to D22S183, recognized by cosmid M56, lies immediately outside the common SRO of the DGS and VCFS deletions, but inside the SRO of the DGS deletions. D22S183 sequences are strongly conserved in primates and weaker hybridizing signals are found in DNA of other mammalian species; no transcripts are however detected in polyA $\mathrm{A}^{+}$RNA from various adult human organs. Probe M51 allows fast reliable screening for $22 \mathrm{q} 11$ deletions using fluorescence in
\end{abstract}

M. P. Mulder $(\bowtie) \cdot$ A. Langeveld · A. Hagemeijer · E. van Drunen Department of Cell Biology and Genetics, Erasmus University, P.O.Box 1738, 3000 DR Rotterdam, The Netherlands

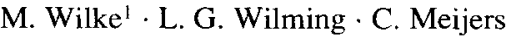

Institute of Paediatric Surgery, Erasmus University, P.O.Box 1738, 3000 DR Rotterdam, The Netherlands

E. C. Zwarthoff · P. H. J. Riegman

Department of Pathology, Erasmus University,

P.O.Box 1738, 3000 DR Rotterdam, The Netherlands

W. H. Deelen - A. M. W. van den Ouweland - D. J. J. Halley Department of Clinical Genetics, University Hospital Dijkzigt, Erasmus University, P.O.Box 1738, 3000 DR Rotterdam, The Netherlands

Present address:

${ }^{1}$ Department of Paediatrics, Sophia Children's Hospital,

3000 DR Rotterdam, The Netherlands situ hybridization. A deletion was found in 11 out of 12 DGS patients and in 3 out of 7 VCFS patients. Two patients inherited the deletion from a parent with mild (atypical) symptoms.

\section{Introduction}

Hemizygosity for a region of chromosome $22 q 11$ has been associated with a range of congenital defects that have recently been brought together under the acronym CATCH22, denoting cardiac defects, abnormal facies, thymic hypoplasia, cleft palate, hypocalcaemia, chromosome 22 (Wilson et al. 1993). The congenital defects include DiGeorge syndrome (DGS), velo-cardio-facial syndrome (VCFS), conotruncal anomaly face (CTAF) syndrome and non-syndromic conotruncal cardiac defects. The common embryonic origin for all these malformations could be a disturbance in the development of mesenchymal derivatives of the hindbrain neural crest (Bockman and Kirby 1984).

DGS, which may be considered to lie at the severe end of the clinical spectrum of $\mathrm{CATCH} 22$, is characterized by hypoplasia/aplasia of the thymus and parathyroid glands, conotruncal heart defect and varying craniofacial dysmorphologies (Conley et al. 1979). Characteristic features of VCFS include cleft palate, typical facies, cardiac defects and learning disabilities (Shprintzen et al. 1978; Goldberg et al. 1993). There is a considerable phenotypic overlap between DGS and VCFS (Goldberg et al. 1985; Stevens et al. 1990). The CTAF syndrome may be identical to VCFS. Most DGS cases appear to be sporadic, but some familial cases have been reported. An autosomal dominant mode of inheritance has been demonstrated for VCFS (Shprintzen et al. 1981; Williams et al. 1985). Although the aetiology of DGS is presumed to be heterogeneous (Lammer and Opitz 1986), recent evidence indicates that chromosome abnormalities involving a region in $22 \mathrm{q} 11$ are a frequent cause of both DGS and VCFS. Cytogenetic abnormalities have been observed in 15-25\% of reported DGS cases, and most of these are either unbalanced translocations resulting in monosomy for the region $22 \mathrm{q} 11$-pter or 
interstitial deletions within 22q11 (Greenberg et al. 1988; Wilson et al. 1992a). Molecular studies using probes for various loci in the $22 q 11$ region have detected submicroscopic deletions in more than $95 \%$ of DGS cases without cytogenetic abnormalities (Scambler et al. 1991; Driscroll et al. 1992a; Carey et al. 1992). Similar deletions have also been demonstrated for a high percentage of VCFS (Scambler et al. 1992; Driscroll et al. 1992b; Kelly et al. 1993) and CTAF cases (Burn et al. 1993). The frequency of deletions is lower if patients referred from a large number of practising clinicians are examined and consequently the diagnostic criteria are less strict (Driscroll et al. 1993). Finally, 22q11 deletions have been detected in a minority of non-syndromic patients with congenital conotruncal cardiac defects (Wilson et al. 1992b; Goldmuntz et al. 1993).

Although the interstitial deletions in most patients appear to be large at the DNA level (>2 Mb), molecular studies have begun to narrow down the smallest region of overlap (SRO) of deletions (Scambler et al. 1991; Driscroll et al. 1992a; Desmaze et al. 1993a; Lindsay et al. 1993; Halford et al. 1993c). At present, it is not known whether DGS/VCFS is caused by deletion of a single gene or whether it is a so-called contiguous gene syndrome, with different extents of the deletions accounting for phenotypic variability. Although the saturation cloning of the SRO is progressing and one candidate gene, TUPLE1, for CATCH22 has recently been identified (Halford et al. $1993 \mathrm{c}$ ), additional genes will have to be cloned in order to elucidate this issue. However, one DGS case has been reported with an apparently balanced translocation $t(2 ; 22)$ (Augusseau et al. 1986). The breakpoint at 22q11 in this case maps within the SRO (Lindsay et al. 1993) but does not disrupt TUPLE1. Together, these data suggest that haplo-insufficiency for a locus or loci in a DiGeorge critical region at $22 \mathrm{q} 11$ can cause multiple congenital malformations. The critical region should lie within the SRO that has been narrowed to an interval of around $500 \mathrm{~kb}$. In most CATCH22 patients, however, particularly in DiGeorge patients, the deletion is larger than $2 \mathrm{Mb}$ (Halford et al. $1993 \mathrm{c})$. Therefore, the chromosomal regions adjacent to the SRO may harbour genes that contribute to the clinical phenotype.

Here, we report the regional localization of the anonymous single-copy polymorphic marker D22S183. By using a cosmid probe and fluorescence in situ hybridization (FISH), we show that this locus maps within the most recently described SRO of the CATCH22 deletions, viz. between TUPLE1 and the locus D22S75.

\section{Materials and methods}

Probes and genomic library screening

NB84 is a $1.0-\mathrm{kb}$ plasmid probe for locus D22S183, which has been assigned to the region 22pter-q1 1 (Van Biezen et al. 1993). It recognizes a $P_{\text {stl }}$ polymorphism (Lekanne Deprez et al. 1991). Plasmid HP500 (Carey ef al. 1992) and cosmids sc4.1 (Carey et al 1992) and scll.1 (Halford et al. 1993a) were kindly provided by
Dr. P.J. Scambler, and $\cos 39$ (Aubry et al. 1993) by Dr. M. Aubry. A cosmid for the locus D22S75 (N25) (Driscroll et al. 1993) was purchased from Oncor (Gaithersburg, Md.). This probe is supplied as digoxigenin-labelled DNA by the manufacturer and is premixed with a digoxigenin-labelled control cosmid ( $\mathrm{pH} 17$ ) for the locus D22S39 in 22q13.3. Probes for the human $\beta$-globin gene and the immunoglobulin $\lambda$ light chain complex were used as controls in Southem hybridizations.

The chromosome-22-specific cosmid library L22NCO31"N" used in this work was constructed at the Biomedical Sciences Division, Lawrence Livermore National Laboratory, Livermore, CA 94550 , USA, under the auspices of the National Laboratory Gene Library Project sponsored by the US Department of Energy (kindly provided by Dr. P. de Jong). Cosmids M5I (33D9) and M56 (38A6) were isolated from this library using NB84 as the probe. Cosmids M69 (87D4) and M78 (114B1) were isolated using a plasmid containing a fragment of the PDGFB gene. These were used as chromosome-22-specific control cosmid probes. Cosmid $\mathrm{cH} 748$ (48H7) was used as a TUPLE1-specific probe (Halford et al. $1993 \mathrm{c}$ ).

Patients and cell lines

Patients were ascertained through clinical geneticists. Blood samples or skin biopsies for the establishment of fibroblast cultures were obtained from propositi and, in some cases, their parents.

Cell lines GM03479, GM05878 and GM00980 were obtained from the NIGMS Human Genetic Mutant Cell Repository (Camden, N.J.). GM03479 is from a DGS patient and has no detectable cytogenetic abnormality (Scambler et al. 1991). GM05878 is from the unaffected father of a DGS child and has a balanced translocation $\mathrm{t}(10 ; 22)(\mathrm{q} 26.3 ; \mathrm{q} 11.2)$ (Cannizzaro and Emanuel 1985). GM00980 is from a VCFS patient with a translocation $t(11 ; 22)$ (q25;q11) and monosomy 22pter-q11 (Fu et al. 1976). ADU is from a DGS patient with an apparently balanced translocation t(2;22)(q14.1;q11.1) (Augusseau et al. 1986) and was kindly provided by Dr. F. Amblard. Hybrid cell lines were established by fusion of fibroblasts from DGS patients with thymidine-kinase-deficient Chinese hamster cells (A3) followed by selection of colonies in HAT culture medium according to standard procedures.

\section{Karyotyping and FISH}

Cells were harvested from cultures of phytohaemagglutinin-stimulated lymphocytes, skin fibroblasts or established cell lines and spread onto slides for the production of $\mathrm{G}$-banded or R-banded chromosome preparations and FISH. In some experiments, confluent fibroblast cultures were enriched for $G_{1}$ interphase cells by growth in medium with $2 \%$ fetal calf serum for 4 days.

FISH was carried out as described by Amoldus et al. (1990) with minor modifications. Cosmid probes were labelled by nick translation with either biotin-16-dUTP or digoxigenin-11-dUTP. The hybridization mixture contained $50 \mathrm{ng}$ of each labelled cosmid plus $1 \mu \mathrm{g}$ human genomic DNA as a competitor in $10 \mu 170 \%$ formamide, $2 \times \mathrm{SSC}(1 \times \mathrm{SSC}=150 \mathrm{mM} \mathrm{NaCl} / 15 \mathrm{mM}$ sodium citrate, $\mathrm{pH} 7.0$ ). After hybridization and washes, the slides were stained in three consecutive steps: (1) avidin-D-fluorescein, (2) biotinylated goat anti-avidin plus sheep anti-digoxigenin-rhodamine, (3) avidin-D-fluorescein plus donkey anti-sheep-Texas Red. The slides were finally embedded in a glycerol mixture containing an antifade reagent and 4,6-diamino-2-phenyl-indole (DAPI) for counterstaining. In case of single-colour labelling (fluorescein), propidium iodide was used as the counterstain. The slides were analysed with a Zeiss Axioskop epifluorescence microscope, using various filter combinations (single, double and triple band).

For $22 q 11$ deletion analysis, metaphase spreads or $\mathrm{G}_{1}$ interphase cells were hybridized simultaneously with the test probe (M51) and a chromosome-22-specific control probe (M69 or M78). The probes were usually stained in two colours but single colour staining was also used for metaphase preparations. 
Metaphases (at least 15 per case) in which both chromosomes 22 gave a signal with the control probe were scored for M51 signals in $22 \mathrm{q} 11$. Alternatively, interphase nuclei (at least 50 per case) with two signals for the control probe were scored for the number of M51 signals. In preparations of normal controls 95-100\% of the metaphases and $90-95 \%$ of the interphase nuclei gave a double (dizygous) signal for M51, indicating the high hybridization efficiency of both M51 and the control probe.

\section{Southern analysis}

Genomic DNA, extracted from blood samples, cultured fibroblasts or established cell lines, was digested with restriction enzymes as recommended by the manufacturer (Boehringer, Mannheim, Germany), separated by agarose gel electrophoresis and transferred to Hybond $\mathrm{N}^{+}$filters (Amersham, UK). DNA probes were labelled with $\left[\alpha^{32} \mathrm{P}\right] \mathrm{dATP}$ and $\left[\alpha^{32} \mathrm{P}\right] \mathrm{dCTP}$ by using the random-primer method.

A Zoo-blot, containing EcoRI-digested genomic DNA from human, Rhesus monkey, Sprague-Dawley rat, BALB/c mouse, dog, cow, rabbit, chicken and Saccharomyces cerevisiae yeast, was purchased from Clontech (Palo Alto, Calif.) and hybridized according to the manufacturer's instructions (hybridization at $65^{\circ}$ $\mathrm{C}$, wash in $2 \times \mathrm{SSC}$ at $60^{\circ} \mathrm{C}$ ).

\section{Results}

\section{D22S183 maps in the commonly deleted region}

To facilitate the screening of probes for their possible location in the commonly deleted region, we generated somatic cell hybrids by fusing thymidine-kinase-deficient Chinese hamster cells with fibroblasts of two unrelated DGS patients both carrying a cytogenetic microdeletion in $22 q 11$. Hybrid clones retaining one chromosome 22 were investigated for the presence of the locus D22S134, by using either the marker probe HP500 (Southern hybridization) or the corresponding cosmid sc4.1 (FISH). This locus is hemizygous in more than $95 \%$ of cytogenetically normal DGS patients (Carey et al. 1992). In this way, hybrid clones containing either the normal or the deleted chromosome 22 were identified. DNA isolated from these hybrids was investigated using Southern blot analysis with probes from various single-copy anonymous markers that have recently been assigned to the region 22pter-q11 (Van Biezen et al. 1993). One of the markers tested, NB84 (locus D22S183), appeared to map within the 22q11 deletion of both DGS patients whose fibroblasts were used to generate hybrid cell lines (Fig. 1). We therefore decided to investigate the map position of this locus and the applicability of plasmid and cosmid probes in Southern and FISH analysis for the routine detection of deletions in CATCH 22 in more detail.

Hybridization of radiolabelled NB84 to a Zoo-blot containing EcoR1-digested DNA from various animal species produced a clear signal only with human and Rhesus monkey DNA (data not shown), leading to the conclusion that D22S183 sequences are conserved in primates. Weakly hybridizing fragments were also detected in other mammalian species (viz. cow, mouse, Chinese hamster). Hybridization of Northern blots containing poly- $\mathrm{A}^{+} \mathrm{RNA}$ from various adult human organs and from

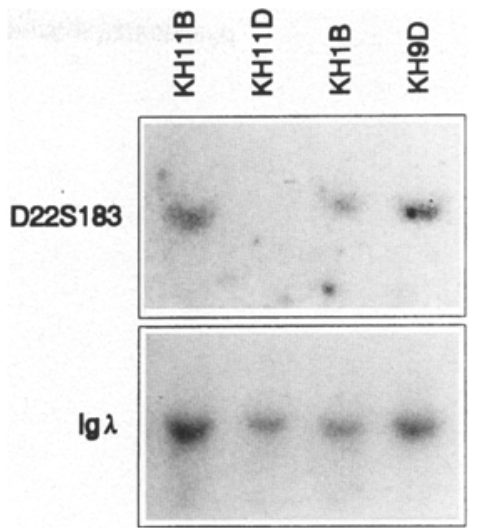

Fig. 1 Autoradiogram of Southern blot of EcoRI-digested DNA from human (DGS)-Chinese hamster hybrid cell lines hybridized sequentially with probe NB84 (D22S183) and a probe from the immunoglobulin $\lambda$ light chain complex (Ig $\lambda)$ as a chromosome-22-specific control. Cytogenetic analysis had shown one chromosome 22 in each hybrid line. Hybrid KH1 1D carries the deleted chromosome 22 ; the other hybrids have retained the normal chromosome 22

a fetus did not result in a signal, indicating that D22S183 is probably not part of an expressed sequence.

\section{Isolation of cosmids M51 and M56}

Screening of the chromosome-22-specific gridded cosmid library L22NC031"N" with NB84 yielded three overlapping cosmids that were suitable for FISH (19B9, 33D9, 83C5). All three mapped to band 22q11 in metaphase chromosomes (Fig. 2a) and were hemizygous in the DGS patients mentioned above. In normal control interphase nuclei in $\mathrm{G}_{1}$ phase, each cosmid produced two hybridization signals. One of the cosmids, 33D9 (lab name M51), was used in further studies. Upon screening the chromosome-22-specific library with NB84, a fourth weakly positive cosmid clone, 38A6 (lab name M56), was identified that was not contiguous with the other cosmids. In situ hybridization of M56 on normal metaphase chromosomes gave a clear signal in 22q11, overlapping with M51. In interphase nuclei, however, M56 produced a signal close to, but separate from, the M51 signal, indicating that the two cosmids recognize different loci.

Cosmid M51 maps within the SRO of CATCH22 deletions and M56 is commonly deleted in DGS patients

For a more detailed regional mapping of M51 and M56, we used the translocation cell lines ADU, GM05878 and GM00980. ADU is from a DGS patient with an apparently balanced translocation $t(2 ; 22)(\mathrm{q} 14.1 ; \mathrm{q} 11.1)$ (Augusseau et al. 1986). Cell line GM05878 contains a balanced translocation $\mathrm{t}(10 ; 22)(\mathrm{q} 26.3 ; \mathrm{q} 11.2)$ and is from the unaffected father of a DGS child (Cannizzaro and Emanuel 1985). The child had monosomy 22pter-q11 (Kelley et al. 1982). FISH analysis showed both M51 and 


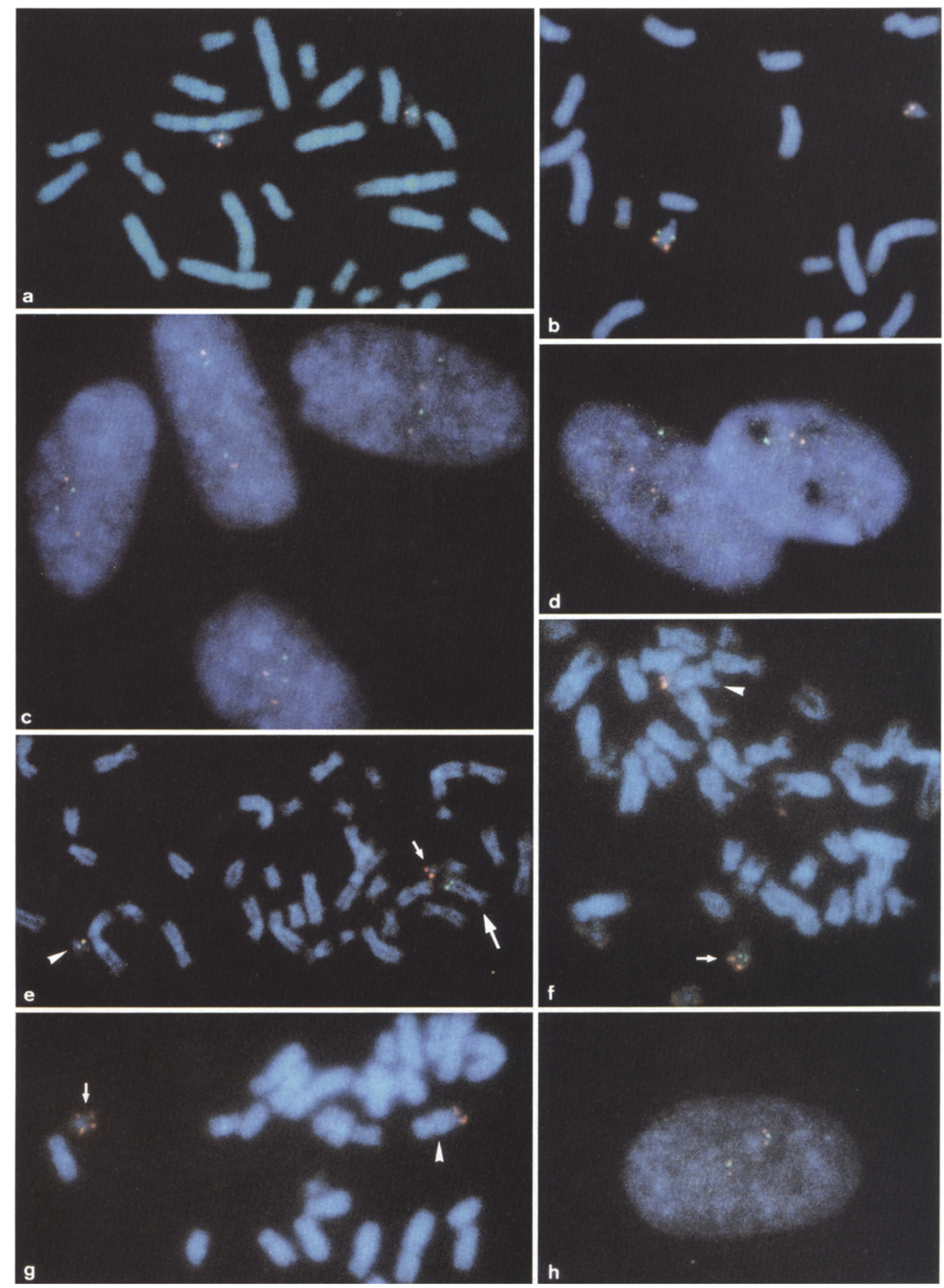


Fig. 2a-h FISH analysis of metaphase chromosomes and $\mathrm{G}_{1}$ interphase nuclei. Direct photomicrographs, without digital enhancement. Probes are coloured either in green (fluorescein) or in red (rhodamine/Texas Red) and DNA is counterstained in blue with DAPI. Fluorescence was registered using a triple-band filterset. a Partial metaphase spread from lymphocyte culture of normal control subject, hybridized with cosmid M51 (D22S183) labelled in red. Both chromosomes 22 show a signal in the q11 region. b Partial metaphase spread from a lymphocyte culture of a DGS patient, hybridized with M51 (green) and the control probe M78 (red). M78 produces a signal on both chromosomes 22 (at q13), but M51 on only one, indicating hemizygosity. $\mathbf{c}, \mathbf{d ~}_{1}$ interphase nuclei from fibroblast cultures of a normal control subject (c) and a DGS patient (d), hybridized with M51 (green) and the chromosome-22specific control probe M78 (red). Both M78 and M51 produce two signals per nucleus in the control (c), whereas in the DGS nuclei (d), M78 produces two signals, but M51 only one. e Partial metaphase spread from the balanced translocation cell line GM05878, hybridized with M51 (red) and sc4.1 (green). In the normal chromosome 22 (arrowhead), the signals of M51 and sc4.1 overlap, resulting in a white signal. The derivative 22 (small arrow) shows a red signal for M51 and the derivative 10 (large arrow) a green signal for sc4.1, indicating that the translocation breakpoint is between the two loci. f Partial metaphase spread from the unbalanced translocation cell line GM00980, hybridized with M51 (green) and M78 (red). The normal chromosome 22 (arrow) shows proximal and distal signals for M51 and M78, respectively. The derivative 11 (arrowhead) shows a signal for M78 but not for M51, indicating that M51 is deleted and maps centromeric to the translocation breakpoint in this cell line. $g$ Partial metaphase spread from cell line GM00980, hybridized with N25 and the control probe $\mathrm{pH} 17$, both in red (the two probes were supplied premixed by the manufacturer, both labelled with digoxigenin, making two-colour hybridization impossible). The normal chromosome 22 (arrow) shows proximal and distal signals for N25 and $\mathrm{pH} 17$, respectively. On the derivative 11 (arrowhead), only the distal signal is present, indicating that N25 is deleted and maps centromeric to the translocation breakpoint. $h \mathrm{G}_{1}$ interphase nucleus from the fibroblast culture of a normal control subject. Triple hybridization with cosmids $\mathrm{cH} 748$ (red), M51 (green) and sc4.1 $(r e d)$. Both triplets show the order red-green-red. In one triplet, the green signal partially overlaps one of the red signals

M56 to be located between the two translocation breakpoints in $22 \mathrm{q} 11$, i.e. telomeric to the ADU breakpoint and centromeric to the GM05878 breakpoint. An example is presented of a GM05878 metaphase cohybridized with cosmids M51 and sc4.1 (Fig. 2e). In agreement with Lindsay et al. (1993), sc4.1 was found to map telomeric to both the ADU and the GM05878 breakpoint and is therefore telomeric to M51 and M56.

Cell line GM00980 is from a VCFS patient and has an unbalanced translocation $\mathrm{t}(11 ; 22)(\mathrm{q} 25 ; \mathrm{q} 11)$ with deletion 22pter-q11 (Fu et al. 1976). According to Lindsay et al. (1993), the breakpoints in GM00980 and GM05878 are close. By using FISH analysis on interphase nuclei and metaphase spreads of GM00980, we have found M51 to be hemizygous, i.e. absent from the derivative 11 translocation chromosome (Fig. 2f), whereas M56 is dizygous. This result places the GM00980 breakpoint between M51 and M56 and shows that M56 is telomeric to M51. Together these data lead to the following order: cen-ADU breakpoint-M51(D22S183)-GM00980 breakpoint-M56GM05878 breakpoint- tel (see also Fig. 5).
Position of TUPLE1, D22S75 (N25) and ZNF74 relative to M51 and M56

The recently described gene TUPLE1 (Halford et al. 1993c) maps between the ADU and GM00980 breakpoints, i.e. in the same region as M51. A cosmid probe for TUPLE1 is $\mathrm{cH} 748$. Two-colour FISH of interphase nuclei showed the hybridization signals of $\mathrm{cH} 748$ and M51 to be frequently overlapping $(47 \%, n=212)$, indicating that the two loci are close. The centromeric-telomeric orientation of M51 and $\mathrm{cH} 748$ was determined by two-colour FISH, as described by Trask et al. (1991), by using sc4.1 as a distal reference point. In this experiment, cells synchronized in $\mathrm{G}_{1}$ were hybridized simultaneously with M51 coloured green and with $\mathrm{cH} 748$ and $\mathrm{sc} 4.1$ both coloured red. The order of the probes was then established by scoring the position of the green probe either between or outside the two red probes. Only nuclei in which the three fluorescent spots were on a straight or obtusely angled line were scored. In the majority of nuclei $(73 \%, n=121)$, the order was red-green-red (Fig. 2h), suggesting that M51 is telomeric to $\mathrm{CH} 748$.

Driscoll et al. (1992a, b, 1993) have described the locus D22S75 (probe N25) as being deleted in the great majority of DGS and VCFS patients. They have mapped this locus centromeric to the GM05878 breakpoint. Using a commercially available digoxigenin-labelled N25 probe, we have investigated the map position of the locus in more detail. FISH analysis of metaphases of the ADU and GM00980 cell lines showed N25 to map between the two translocation breakpoints (Fig. $2 \mathrm{~g}$ ), as do M51 and $\mathrm{cH} 748$ (TUPLE1). The N25 probe is supplied pre-mixed with a digoxigenin-labelled control probe for a locus at 22q13.3. This locus is too far away to be used as a distal reference point in two-colour FISH analysis of $\mathrm{G}_{1}$ interphase nuclei directed at determining the centromeric-telomeric orientation of N25 and M51. Therefore, M56 was used as a reference marker. $G_{1}$ interphase nuclei were hybridized simultaneously with M51 and M56 coloured green and the N25 mix coloured red. Nuclei were selected in which the red signals (for N25 and the locus at 22q13.3) were sufficiently separate and in which two green signals (for M51 and M56) were close to one red signal. The position of this red signal was then scored either between or outside the two green signals. In $64 \%$ of the cases $(n=158)$, the red signal was between the green signals, indicating that N25 is telomeric to M51. This conclusion was also supported by the frequencies of overlapping hybridization signals in interphase nuclei hybridized with either N25 and M51 (21\% overlap, $n=178$ ) or N25 and M56 (35\% overlap, $n=118$ ). Knowing that both N25 and M51 are centromeric to M56, these overlap frequencies indicate that N25 is telomeric to M51.

Aubry et al. (1993) have shown that the zinc finger gene ZNF74 maps to 22q11 and is consistently deleted in DGS patients. Using a cosmid probe $(\cos 39)$, we have investigated, by FISH, the position of this gene relative to the translocation breakpoints of ADU, GM00980 and GM05878. In all three cell lines, ZNF74 mapped telom- 
eric to the translocation breakpoint in $22 \mathrm{q} 11$. The position of ZNF74 was then established relative to the sc11.1B locus (D22S139). Cosmid sc11.1 (Halford et al. 1993a) recognizes two loci, sc11.1A and sc11.1B, that are approximately $2 \mathrm{Mb}$ apart and that are commonly deleted together in DGS patients (Desmaze et al. 1993b; Lindsay et al. 1993). Locus sc11.1A is centromeric to the ADU breakpoint and sc11.1B is telomeric to the GM05878 breakpoint. The hybridization signals for the two loci are not distinguishable on metaphase chromosomes but are separate in interphase nuclei. To establish whether ZNF74 is between or outside the sc 11.1 loci, $\mathrm{G}_{1}$ interphase nuclei were hybridized simultaneously with $\cos 39$ coloured green and sc 11.1 coloured red and the order of the probes was scored. In $65 \%$ of the cases $(n=203)$, the order was red-red-green, suggesting that ZNF74 is telomeric to sc11.1B. The distance between these loci can be no more than a few hundred kilobase pairs, since there was a frequent overlap between the green signal and one of the red signals.

\section{CATCH22 patient analysis}

Hemizygosity assays were performed using blood samples and/or skin fibroblast cultures of 12 DGS and seven VCFS patients and, in some cases, their parents. Cytogenetic analysis revealed a microdeletion in three DGS cases. All other subjects were karyotypically normal. In addition to the patient material collected in Rotterdam, we also analysed the previously described cell line GM03479, which has been derived from a DGS patient but in which, so far, no hemizygosity has been detected (Scambler et al. 1991).

Hemizygosity for the locus D22S183 was studied in three different ways: restriction fragment length polymorphism (RFLP) analysis, quantitative Southern analysis and FISH. In Southern analysis of PstI-digested genomic DNA, a polymorphism recognized by NB84 is in some cases informative for the detection of deletions (Fig. 3). Two allelic bands of 6.0 and $4.7 \mathrm{~kb}$ can be detected (the sizes of the PstI fragments have previously been incor-

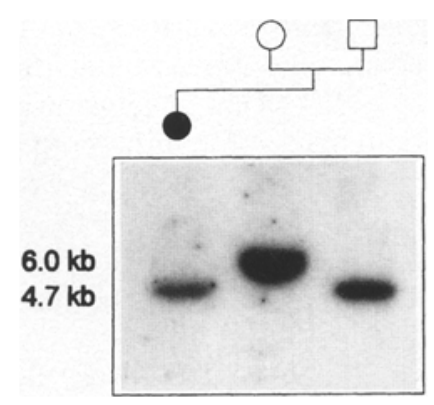

Fig. 3 Autoradiogram of Southern blot of Pst I-digested DNA from a proband and her parents, hybridized with probe NB84 (locus D22S183), which reveals a polymorphism in this family. The mother and father are homozygous for the $4.6-\mathrm{kb}$ and $4.0-\mathrm{kb}$ allele, respectively. The proband failed to inherit a maternal allele

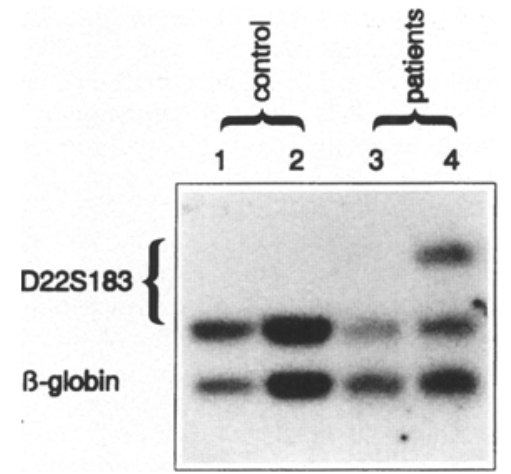

Fig. 4 Quantitative Southern analysis of PstI-digested DNA from two patients (lanes 3,4 ) and a control subject (lanes 1, 2; in lane 2 , a double amount of DNA was loaded). The blot was hybridized simultaneously with probe NB84 (locus D22S183) and a probe for the $\beta$-globin locus as a control. Comparison of the signal intensities produced by the test probe and the control probe shows that one patient (lane 3), who was diagnosed as having VCFS, is hemizygous for D22S183. The other patient (lane 4) is heterozygous for the $P_{s t I}$ polymorphism in D22S183 and therefore has no deletion at this locus. This patient has congenital heart disease and minor facial dysmorphisms

rectly assessed at 4.6 and $4.0 \mathrm{~kb}$; Lekanne Deprez et al. 1991). The estimated frequencies among Caucasians are 0.81 and 0.19 for the $6.0-\mathrm{kb}$ and $4.7-\mathrm{kb}$ bands respectively (Lekanne Deprez et al. 1991). In a series of 38 consecutive patients (DGS, VCFS and non-syndromic congenital heart disease), the Pst $\mathrm{L}$ polymorphism was informative in 12 cases. If no informative polymorphism was found, hemizygosity for D22S183 was studied by quantitative Southern analysis of PstI-digested genomic DNAs. Filters were hybridized simultaneously with radiolabelled NB84 and a probe from the $\beta$-globin gene as a control. The latter probe recognizes a fragment of $4.4 \mathrm{~kb}$, which is in the same range as the NB84 fragments. From the autoradiogram (Fig. 4), the dosage of the D22S183 locus was established by comparing the intensities of the hybridization signals of the two probes.

FISH analysis with the cosmid probe M51 was found to be a rapid reliable hemizygosity assay. Metaphase spreads or preparations of cultures that had been enriched for $G_{1}$ interphase cells were simultaneously hybridized with M51 and a chromosome-22-specific control cosmid corresponding to the PDGFB locus at 22q13.1 (Fig. 2b-d). In case of a deletion, $100 \%$ of the metaphases and $97-100 \%$ of the interphase nuclei that revealed a double (dizygous) signal with the control probe were scored as hemizygous for M51.

Eleven out of 12 DGS patients and 3 out of 7 VCFS patients were hemizygous for the D22S183 locus. In all cases, Southern and FISH analyses led to identical conclusions. In view of speed and reliability of diagnosis, FISH using cosmid M51 is the method of choice for the detection of $22 q 11$ deletions. Two DGS patients had inherited the deletion from a parent. The hemizygous mother of one patient had been operated for tetralogy of Fallot at the age of 6 years. The other patient inherited the 
Table 1 Clinical features of four VCFS patients and one DGS patient without detectable $22 \mathrm{q} 11$ deletion (+ presence of clinical trait, - absence of clinical trait, nd no data available, $(a)$ mother and daughter, $(b)$ sister has VCFS dysmorphic features but no cardiac defect, $(c)$ mother has prominent nose, cardiac murmur, $(d)$ sib has mental retardation, dysmorphic face, deafness)

\begin{tabular}{llllll}
\hline & VCF-Ro2 & VCF-Ro3 & VCF-Ro5 & VCF-Ro6 & DG-Ro9 \\
\hline Cardiac defects & + & + & + & + & + \\
Typical facies & + & + & + & + & + \\
Palatal abnormalities & nd & nd & + & + & + \\
Learning disabilities & nd & nd & + & nd & nd \\
Clinodactyly & nd & + & + & + & - \\
Decreased lymphoid tissue & nd & nd & nd & nd & + \\
Other & (a) & (a) & (b) & (c) & (d) \\
\hline
\end{tabular}

deletion from her father, who suffers from psychiatric illness but has no physical abnormalities. To obtain an indication of the size of the deletions, FISH analysis was also performed with the cosmid probes sc4.1 and sc11.1. All cases hemizygous for D22S183 (patients and parents) were also hemizygous for sc4.1 and for both sc11.1 loci (A and B), suggesting that the deletions span at least $2 \mathrm{Mb}$.

One DGS case, four VCFS cases and the cell line GM03479 were dizygous for D22S183 (clinical features of these patients are given in Table 1). No hemizygosity for sc4.1, sc11.1 or the TUPLE1 cosmid $\mathrm{cH} 748$ was detected in the DGS patient and one VCFS patient.

\section{Discussion}

An SRO of 22q11 deletions associated with DGS and VCFS has been delineated in several recent publications (Driscroll et al. 1992a; Desmaze et al. 1993a; Lindsay et al. 1993; Halford et al. 1993c). It has not been possible to narrow the SRO to an interval smaller than approximately $500 \mathrm{~kb}$, suggesting that $\mathrm{CATCH} 22$ might be a contiguous gene syndrome.

By using the cosmid probe M51, we have determined the map position of the anonymous marker locus D22S183 relative to a number of translocation breakpoints and other known loci. D22S183 maps within the SRO. A second locus, recognized by cosmid M56, which contains sequences with weak homology to D22S183, is located more distally, just outside the SRO. A positional map is presented in Fig. 5. Recently, Halford et al. (1993c) have described the gene TUPLE1, which encodes a putative transcriptional regulator and which is located close to (on the distal side of) the balanced $t(2 ; 22)$ translocation breakpoint in the DGS patient ADU. Although there is no indication yet that the gene is disrupted or that its expression is disturbed by the ADU translocation, it is reasonable to suppose that haplo-insufficiency for TUPLE1 contributes to the malformations in DGS and VCFS. Another locus of interest, D22S75 (probe N25), is in the SRO of the DGS and VCFS deletions described by Driscoll et al (1992a). D22S75 is adjacent to a NotI site that is located in an HTF island (McDermid et al. 1989) and may therefore be at the 5' end of a gene. In the present investigation, it is shown that D22S75 is between the ADU and GM00980 breakpoints and therefore within the SRO described by Halford et al. (1993c). The order of markers in the SRO was determined to be TUPLE1 D22S183 - D22S75 (Fig. 5), the TUPLE1-D22S183 interval being shorter than the D22S183-D22S75 interval. According to Halford et al. (1993c), the interval between TUPLE1 and the GM00980 breakpoint should be less than $275 \mathrm{~kb}$. We therefore estimate D22S75 to be close to this breakpoint and D22S183 to be less than $200 \mathrm{~kb}$ from TUPLE1. In the absence of evidence that deletion or mutation of TUPLE1 is sufficient to cause $\mathrm{CATCH} 22$, the identification of other genes in the SRO remains an im-

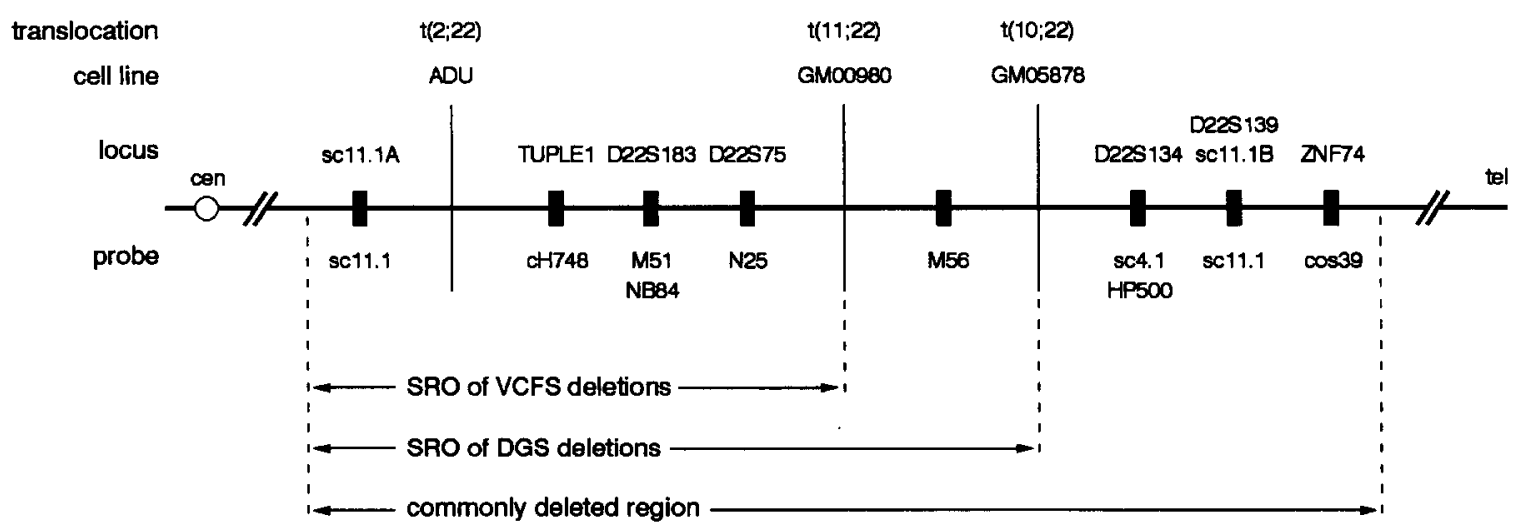

Fig. 5 Diagram of the DiGeorge chromosomal region at $22 q 11$ showing the relative order of loci and translocation breakpoints studied. Vertical lines Position of translocation breakpoints, rec- tangles loci (genes). The probes (cosmids and plasmids) used in the present study are indicated for each locus. Chromosomal distances have not been drawn to scale 
portant issue. In this respect, D22S75 and D22S183 may be relevant loci.

The region that is commonly deleted in the majority of patients is considerably larger than the SRO. Most $\mathrm{CATCH} 22$ deletions extend over a distance of more than $2 \mathrm{Mb}$, usually including the sc11.1B locus (Desmaze et al. 1993a, b; Halford et al. 1993c), as was also shown in our patient series. However, there is some indication that a less distal extension of a deletion is more likely to be associated with VCFS than with DGS. According to Halford et al. (1993c), a deletion not including the scll.1B locus has been found in 7 out of 91 DGS and 6 out of 16 VCFS cases (in their Table 1, the patient with deletion C1/79-sc11.1A is the child of GM05878 and the patient with deletion scF5-sc11.1A is the GM0980 case; in the Table, these should be interchanged as DGS and VCFS, respectively; personal communication Dr. P.J. Scambler). In six of the seven DGS cases, the deletion includes the D22S134 (sc4.1) locus, whereas in the six VCFS cases, the deletion does not extend distally to this locus. The only DGS patient who probably had a deletion not extending so far distally is the child of GM05878. For VCFS, the most centromeric distal deletion boundary is found in GM00980. This means that haplo-insufficiency for a gene or genes in the region between the GM00980 and GM05878 breakpoints, and perhaps also in the adjacent distal region, might play a specific role in the pathogenesis of DGS. The cosmids M56 (present investigation), $\mathrm{Cl} / 79$ (Halford 1993c), and D0832 (Lindsay et al. 1993) could help in finding such genes. Furthermore, genes located more distally but within the commonly deleted region, such as COMT (Dunham et al. 1992; Desmaze et al. 1993a), T10 (Halford et al. 1993b) and the zinc finger gene ZNF74 (Aubry et al. 1993), might contribute to the clinical phenotype in CATCH22 patients. Although ZNF74 was found to be deleted in 23 out of 24 DGS patients (Aubry et al. 1993), our FISH analysis now shows that the gene maps close to, and possibly distal to, sc11.1B, i.e. far outside the SRO.

Family members with apparently identical 22q11 deletions can be either mildly or severely affected. In two of the DGS cases studied here, the deletion was inherited from a parent. In both cases, the parent does not have the DGS or VCFS phenotype. The mother of one patient has been operated on for tetralogy of Fallot. The father of the other patient suffers from psychiatric illness but has no physical complaints. Inheritance of a $22 \mathrm{q} 11$ deletion with increased severity of the clinical phenotype in the second generation has been reported (Wilson et al. 1991, 1992b; Desmaze et al. 1993a; Driscroll et al. 1993). Our findings once more indicate that, if an individual with isolated conotruncal heart defect is found to have a $22 \mathrm{q} 11$ deletion, there is a risk of more complicated malformations in the offspring.

A small number of DGS and a larger number of VCFS patients have been described in whom no hemizygosity was found for any of the loci investigated (Halford et al. 1993c). The prevalence of such cases may depend on the strictness of the diagnostic criteria (Driscroll et al. 1993). Moreover, in our series, one DGS and four VCFS cases, and the cell line GM03479 revealed no hemizygosity. Patient DG-Ro9 (Table 1) has the major features of DGS but he has a dysmorphic mentally retarded brother with no DGS features, indicating that the malformations might have another basis in this family. The VCFS patients included in Table 1 all have the major features of the syndrome. In addition, a genetic aetiology is suggested by the fact that, in all four cases, family members have VCFS dysmorphic features. An explanation could be that either there is a second VCFS locus or the mutation in $22 \mathrm{q} 11$ cannot be detected with our present methods. An indication for a second DGS locus comes from patients with a deletion in 10p13 (Greenberg et al. 1988; Monaco et al. 1991). Alternatively, the disease does not have a genetic basis in the non-deleted cases.

Acknowledgements We thank Bert de Vries, Hanne MeijersHeijboer, Martinus F. Niermeijer, Cees Schaap, and Eveline van Swaay-Wesby for sharing clinical information and making the patient material available. We also thank Gerard Grosveld and Jacques van Dongen for their help in the initial phase of this study. Bep Smit assisted with karyotyping and FISH analysis. We thank Mirko Kuit for assistance with photography and Marike van Geest for typing the manuscript. Hans Galjaard, Frank G. Grosveld and Jan C. Molenaar supported us throughout this work. The study was supported financially by the Erasmus University and by grants from the Sophia Foundation for Medical Research (no. 157) and the European Union (Biomed).

\section{References}

Arnoldus EPJ, Wiegant J, Noordermeer IA, Wessels JW, Beverstock GC, Grosveld GC, Van der Ploeg M, Raap AK (1990) Detection of the Philadelphia chromosome in interphase nuclei. Cytogenet Cell Genet 54:108-111

Aubry M, Demczuk S, Desmaze C, Aikem M, Aurias A, Julien J$P$, Rouleau GA (1993) Isolation of a zinc finger gene consistently deleted in DiGeorge syndrome. Hum Mol Genet 2: $1583-1587$

Augusseau S, Jouk S, Jalbert P, Prieur M (1986) DiGeorge syndrome and 22q11 rearrangements. Hum Genet 74:206

Bockman DE, Kirby ML (1984) Dependence of thymus development on derivatives of the neural crest. Science 223:498-500

Burn J, Takao A, Wilson D, Cross I, Momma K, Wadey R, Scambler P, Goodship J (1993) Conotruncal anomaly face syndrome is associated with a deletion within chromosome $22 \mathrm{q} 11 . \mathrm{J}$ Med Genet $30: 822-824$

Cannizzaro LA, Emanuel BS (1985) In situ hybridization and translocation breakpoint mapping. III. DiGeorge syndrome with partial monosomy of chromosome 22. Cytogenet Cell Genet 39:179-183

Carey AH, Kelly D, Halford S, Wadey R, Wilson D, Goodship J, Burn J, Paul T, Sharkey A, Dumanski J, Nordenskjold M, Williamson R, Scambler PJ (1992) Molecular genetic study of the frequency of monosomy $22 \mathrm{q} 11$ in DiGeorge syndrome. Am J Hum Genet 51:964-970

Conley ME, Beckwith JB, Mancer JFK, Tenckhoff L (1979) The spectrum of the DiGeorge syndrome. J Pediatr 94:883-890

Desmaze C, Prieur M, Amblard F, Aikem M, LeDeist F, Demczuk S, Zucman J, Plougastel B, Delattre O, Croquette M-F, Brevière G-M, Huon C, Le Merrer M, Mathieu M, Sidi D, Stephan J-L, Aurias A (1993a) Physical mapping by FISH of the DiGeorge critical region (DGCR): involvement of the region in familial cases. Am J Hum Genet 53:1239-1249

Desmaze C, Scambler P, Prieur M, Halford S, Sidi D, Le Deist F, Aurias A (1993b) Routine diagnosis of DiGeorge syndrome by fluorescent in situ hybridization. Hum Genet 90:663-665 
Driscroll DA, Budarf ML, Emanuel BS (1992a) A genetic etiology for DiGeorge syndrome: consistent deletions and microdeletions of 22q11. Am J Hum Genet 50:924-933

Driscroll DA, Spinner NA, Budarf ML, McDonald-McGinn DM, Zackai EH, Goldberg RB, Shprintzen RJ, Saal HM, Zonana J, Jones MC, Mascarello JT, Emanuel BS (1992b) Deletions and microdeletions of $22 \mathrm{q} 11.2$ in velo-cardio-facial syndrome. Am J Med Genet 44:261-268

Driscroll DA, Salvin J, Sellinger B, Budarf ML, McDonaldMcGinn DM, Zackai EH, Emanuel BS (1993) Prevalence of 22q11 microdeletions in DiGeorge and velocardiofacial syndromes: implications for genetic counselling and prenatal diagnosis. J Med Genet 30:813-817

Dunham I, Collins J, Wadey R, Scambler P (1992) Possible role for COMT in psychosis associated with velo-cardio-facial syndrome. Lancet 340:1361-1362

Fu W-N, Borgaonkar DS, Ladewig PP, Weaver J, Pomerance HH (1976) Structural aberrations of the long arm of chromosome no. 22. Clin Genet 10:329-336

Goldberg R, Marion R, Borderon M, Wiznia A, Shprintzen RJ (1985) Phenotypic overlap between velo-cardio-facial syndrome (VCF) and the DiGeorge sequence (DGS). Am J Hum Genet 37 [Suppl] : A54

Goldberg R, Motzkin B, Marion R, Scambler PJ, Shprintzen RJ (1993) Velo-cardio-facial syndrome: a review of 120 patients. Am J Med Genet 45:313-319

Goldmuntz E, Driscroll D, Budarf ML, Zackai EH, McDonaldMcGinn DM, Biegel JA, Emanuel BS (1993) Microdeletions of chromosomal region $22 \mathrm{q} 11$ in patients with congenital conotruncal cardiac defects. J Med Genet 30:807-812

Greenberg F, Elder FFB, Haffner P, Northrup H, Ledbetter DH (1988) Cytogenetic findings in a prospective series of patients with DiGeorge anomaly. Am J Hum Genet 43:605-61 1

Halford S, Lindsay E, Nayudu M, Carey AH, Baldini A, Scambler PJ (1993a) Low-copy-number repeat sequences flank the DiGeorge/velo-cardio-facial syndrome loci at 22q11. Hum Mol Genet 2:191-196

Halford S, Wilson DI, Daw SCM, Roberts C, Wadey R, Kamath S, Wickremasinghe A, Burn J, Goodship J, Mattei M-G, Moormon AFM, Scambler PJ (1993b) Isolation of a gene expressed during early embryogenesis from the region of $22 \mathrm{q} 11$ commonly deleted in DiGeorge syndrome. Hum Mol Genet 2: $1577-1582$

Halford S, Wadey R, Roberts C, Daw SCM, Whiting JA, O'Donnell H, Dunham I, Bentley D, Lindsay E, Baldini A, Francis F, Lehrach H, Williamson R, Wilson DI, Goodship J, Cross I, Burn J, Scambler PJ (1993-c) Isolation of a putative transcriptional regulator from the region of $22 \mathrm{q} 11$ deleted in DiGeorge syndrome, Shprintzen syndrome and familial congenital heart disease. Hum Mol Genet 2:2099-2107

Kelley RI, Zackai EH, Emanuel BS, Kistenmacher M, Greenberg F, Punnett HH (1982) The association of the DiGeorge anomalad with partial monosomy of chromosome 22. J Pediatr 101: $197-200$

Kelly D, Goldberg R, Wilson D, Lindsay E, Carey A, Goodship J, Burn J, Cross I, Shprintzen RJ, Scambler PJ (1993) Confirmation that the velo-cardio-facial syndrome is associated with haplo-insufficiency of genes at chromosome $22 \mathrm{q} 11$. Am J Med Genet $45: 308-312$

Lammer EJ, Opitz JM (1986) The DiGeorge anomaly as a developmental field defect. Am J Med Genet Suppl 2:113-127
Lekanne Deprez RH, Van Biezen NA, Heutink P, Boejharat KRGS, De Klein A, Geurts van Kessel AHM, Zwarthoff EC (1991) A new polymorphic probe on chromosome 22: NB84 (D22S183). Nucleic Acids Res 19:687

Lindsay EA, Halford S, Wadey R, Scambler PJ, Baldini A (1993) Molecular cytogenetic characterization of the DiGeorge syndrome region using fluorescence in situ hybridization. Genomics 17:403-407

McDermid HE, Budarf ML, Emanuel BS (1989) Toward a longrange map of human chromosomal band $22 \mathrm{q} 11$. Genomics 5 : $1-8$

Monaco G, Pignata C, Rossi E, Mascellaro O, Cocozza S, Ciccimarra F (1991) DiGeorge anomaly associated with 10p deletion. Am J Med Genet 39:215-216

Scambler PJ, Carey AH, Wyse RKH, Roach S, Dumanski JP, Nordenskjold $M$, Williamson $R$ (1991) Microdeletions within $22 \mathrm{q} 11$ associated with sporadic and familial DiGeorge syndrome. Genomics 10:201-206

Scambler PJ, Kelly D, Lindsay E, Williamson R, Goldberg R, Shprintzen R, Wilson DI, Goodship JA, Cross IE, Burn J (1992) Velo-cardio-facial syndrome associated with chromosome 22 deletions encompassing the DiGeorge locus. Lancet 339:11381139

Shprintzen RJ, Goldberg RB, Lewin ML, Sidoti EJ, Berkman MD, Argamaso RV, Young D (1978) A new syndrome involving cleft palate, cardiac anomalies, typical facies, and learning disabilities: velo-cardio-facial syndrome. Cleft Palate J 5:56-62

Shprintzen RJ, Goldberg RB, Young D, Wolford L (1981) The velo-cardio-facial syndrome: a clinical and genetic analysis. Pediatrics $67: 167-172$

Stevens CA, Carey JC, Shigeoka AO (1990) DiGeorge anomaly and velocardiofacial syndrome. Pediatrics $85: 526-530$

Trask BJ, Massa H, Kenwrick S, Gitschier J (1991) Mapping of human chromosome Xq28 by two-color fluorescence in situ hybridization of DNA sequences to interphase cell nuclei. Am J Hum Genet $48: 1-15$

Van Biezen NA, Lekanne Deprez RH, Thijs A, Heutink P, Oostra BA, Geurts van Kessel AHM, Zwarthoff EC (1993) Isolation and characterization of 25 unique DNA markers for human chromosome 22. Genomics $15: 206-208$

Williams MA, Shprintzen RJ, Goldberg RB (1985) Male-to-male transmission of the velo-cardio-facial syndrome: a case report and review of 60 cases. J Craniofacial Genet Dev Biol 5: $175-180$

Wilson DI, Cross IE, Goodship JA, Coulthard S, Carey AH, Scambler PJ, Bain HH, Hunter AS, Carter PE, Burn J (1991) DiGeorge syndrome with isolated aortic coarctation and isolated ventricular septal defect in three sibs with a $22 \mathrm{q} 11$ deletion of maternal origin. Br Heart $\mathrm{J} 66: 308-312$

Wilson DI, Cross IE, Goodship JA, Brown J, Scambler PJ, Bain HH, Taylor JFN, Walsh K, Bankier A, Burn J, Wolstenholme J (1992a) A prospective cytogenetic study of 36 cases of DiGeorge syndrome. Am J Hum Genet 51:957-963

Wilson DI, Goodship JA, Burn J, Cross IE, Scambler PJ (1992b) Deletions within chromosome $22 \mathrm{q} 1 \mathrm{l}$ in familial congenital heart disease. Lancet 340:573-575

Wilson DI, Burn J, Scambler P, Goodship J (1993) DiGeorge syndrome: part of CATCH22. J Med Genet 30:852-856 